\title{
Learning Desire Is Predicted by Similar Neural Processing of Naturalistic Educational Materials
}

\author{
(1)Yi Zhu (朱怡), ${ }^{1}$ (D) Yafeng Pan (潘亚峰), ${ }^{1,2}$ and Yi Hu (胡谊) ${ }^{1}$
}

https://doi.org/10.1523/ENEURO.0083-19.2019

${ }^{1}$ School of Psychology and Cognitive Science, East China Normal University, Shanghai 200062, People's Republic of China, ${ }^{2}$ Neuropsychology and Functional Neuroimaging Research Unit (UR2NF), ULB Neuroscience Institute (UNI), Université Libre de Bruxelles, 1050 Bruxelles, Belgium

\begin{abstract}
Naturalistic stimuli can elicit highly similar brain activity across viewers. How do naturalistic educational materials engage human brains and evoke learning desire? Here, we presented 15 audiovisual course clips (each lasting $\sim 120$ s) to university students and recorded their neural activity through electroencephalography. Upon finishing all the video viewings, subjects ranked 15 courses in order of learning desire and reported the reasons for high learning desire (i.e., "value" and "interest"). The brain activity during the video viewing was measured as the neural similarity via intersubject correlation (ISC), that is, correlation between each subject's neural responses and those of others. Based on averaged learning desire rankings across subjects, course clips were classified with high versus medium versus low motivational effectiveness. We found that the ISC of high effective course clips was larger than that of low effective ones. The ISC difference (high vs low) was positively associated with subjects' learning desire difference (high vs low). Such an association occurred when viewing time accumulated to $\sim 80 \mathrm{~s}$. Moreover, ISC was correlated with "interest-based" rather than "value-based" learning desire. These findings advance our understanding of learning motivation via the neural similarity in the context of on-line education and provide potential neurophysiological suggestions for pedagogical practices.
\end{abstract}

Key words: electroencephalography (EEG); intersubject correlation (ISC); learning desire; motivational effectiveness; naturalistic stimuli; neural similarity

\section{Significance Statement}

This study shows that naturalistic educational materials with high motivational effectiveness elicit larger neural similarity across learners than less effective ones. Importantly, the neural similarity serves as a sensitive predictor of learners' course-learning desire. It is suggested that the use of an emerging electroencephalography-derived intersubject correlation approach works with evaluating the quality of audiovisual educational materials. Hence, such a novel approach is promising to provide neurophysiological information for pedagogical practices.

\section{Introduction}

Learning desire is an important prerequisite for human learning to occur. How to evoke learning desire is a persistent concern in the field of educational psychology and pedagogy (Todd, 2013). Recently, on-line courses

Received March 8, 2019; accepted July 28, 2019; First published August 19, 2019.

Author contributions: Y.Z., Y.P., and Y.H. designed research; Y.Z. performed research; Y.Z. analyzed data; Y.Z., Y.P., and Y.H. wrote the paper.

The authors declare no competing financial interests. have brought a tremendous transformation to education, as evidenced by their use in many open learning systems, such as Coursera and Khan Academy (Copley, 2007; Waldrop, 2013). Compared with the traditional classroom learners, on-line learners experience lower-level interac-

This work was sponsored by the National Natural Science Foundation of China (Grant 31872783).

Acknowledgments: We thank our subjects for their participation, Yang Liu for assistance in data collection, and Jieqiong Liu for comments on the earlier manuscript. 
tivity and thus are more susceptible to quitting learning or dropping out of courses (Szpunar et al., 2013; Kizilcec et al., 2015). Therefore, evoking learning desire is of great importance, especially in the context of on-line education (Visser, 1998; Keller and Suzuki, 2004). To this end, one good practice is to introduce audiovisual materials during the introductory phase of the course (Grant and Thornton, 2007; Kay, 2012).

Currently, there are two main hypotheses that account for potential factors contributing to learning desire. First, the "value-based" hypothesis (Atkinson, 1957; Eccles et al., 1983) proposes that helping learners perceive the value (e.g., utility value) will effectively promote learning desire. The perceived utility value of courses influences course enrollment decisions (Updegraff et al., 1996; Canning et al., 2018) and academic achievements (Hulleman et al., 2010). Second, the interest-based hypothesis (Krapp, 2002; Hidi and Renninger, 2006), postulates that guiding learners to develop interest will effectively boost learning desire. Interest, as the saying goes, is the best teacher. Interest contributes to learners' further study (Mitchell, 1993; Ainley et al., 2002; Harackiewicz et al., 2002; Renninger and Hidi, 2002; Schiefele, 2009) and improves learning performance (Rotgans and Schmidt, 2014).

In the context of on-line education, learning desire evoked by audiovisual educational materials has been rarely studied from the neural perspective. To decode human brain activity during real-world experiences, previous studies have measured individuals' neural responses to discrete and simplified artificial stimuli; these responses comprise electroencephalography (EEG)derived event-related potentials and functional magnetic resonance imaging (fMRI)-derived blood oxygenation level-dependent (BOLD) signals (Spiers and Maguire, 2007). Beyond all that, emerging neuroscience research has started to measure the neural similarity (i.e., grouplevel similar neural responses) to concrete and complex naturalistic stimuli from a "collective-brain" perspective. Indeed, when exposed to the same stimulus, individual brains tend to tick collectively in synchronized spatiotemporal patterns (Hasson et al., 2004). The neural similarity can be quantified by intersubject correlation (ISC), that is, correlation between individual subject's neural responses and those of others (Cohen et al., 2018). Using the ISC approach, previous fMRI research reveals that movie viewing elicits highly similar neural activity across viewers (Hasson et al., 2004). Within several-minute narratives, time-resolved ISC peaks during the viewing of scenes with high emotional arousal and valence (Hasson et al., 2004; Nummenmaa et al., 2012). Moreover, ISC is indicative of the powerfulness of political speeches (Schmälzle

Correspondence should be addressed to Yi Hu at yhu@psy.ecnu.edu.cn or Yafeng Pan at yfpan.ecnu@gmail.com.

https://doi.org/10.1523/ENEURO.0083-19.2019

Copyright (C) 2019 Zhu et al.

This is an open-access article distributed under the terms of the Creative Commons Attribution 4.0 International license, which permits unrestricted use, distribution and reproduction in any medium provided that the original work is properly attributed. et al., 2015) and the effectiveness of antialcohol public service announcements (Imhof et al., 2017).

Apart from fMRI-derived ISC, previous EEG studies have captured significantly correlated components during the watching of movie clips, TV series, and commercials (Dmochowski et al., 2012, 2014). Correlated components were extracted from multichannel EEG time series to maximize the correlation based on a signal decomposition method (Dmochowski et al., 2012). EEG-derived ISC has been found to indicate attentional engagement during the narrative video viewing (Dmochowski et al., 2012; Cohen et al., 2017) and preferential attitudes toward Super Bowl commercials (Dmochowski et al., 2014). In a recent study, learners were asked to attentively or inattentively watch on-line educational videos, during which their brain activity was measured (Cohen et al., 2018). EEG-derived ISC discriminates between the attentive and inattentive viewings and predicts the learning performance. In a real-world classroom, EEG-derived ISC has also been found to associate with engagement and attentional modulation (Poulsen et al., 2017).

Building upon previous findings, the EEG-derived ISC approach holds the potential to uncover the neural underpinnings during the natural processing of audiovisual educational materials. In current study, we recorded EEG signals while learners were viewing audiovisual course clips. The ISC approach was adopted to examine the neural similarity across learners. Upon finishing all the video viewings, subjects ranked 15 courses in order of learning desire and reported the reasons of high learning desire (i.e., "value" and "interest"). The viewing of course clips was expected to prompt significant neural similarity across learners because brains tend to tick collectively during natural vision (Hasson et al., 2004; Dmochowski et al., 2012). Moreover, considering the potential links from the neural similarity to the effectiveness of naturalistic materials (Schmälzle et al., 2015; Imhof et al., 2017), and subjects' attentional engagement (Dmochowski et al., 2012; Cohen et al., 2017; Poulsen et al., 2017) and preferential attitudes (Dmochowski et al., 2014), we expected that the neural similarity could be indicative of the motivational effectiveness of course clips and serve as a predictor of learning desire. Specifically, we hypothesized that (1) ISC should be higher for course clips ranked with high versus low learning desire, and (2) the ISC difference (high vs low) should be positively correlated with subjects' learning desire difference (high vs low). Finally, to provide neurophysiological suggestions for why some naturalistic educational materials elicited high learning desire, we explored the association between ISC and potential reasons [e.g., value (Hulleman et al., 2008) and interest (Harackiewicz et al., 2002)].

\section{Materials and Methods}

\section{Subjects}

Fifteen subjects (three males; mean age, 21 years; age range, 18-25 years) were recruited through public announcement at the East China Normal University (Shanghai, People's Republic of China). All of them were right handed and in good health, and had normal or corrected- 


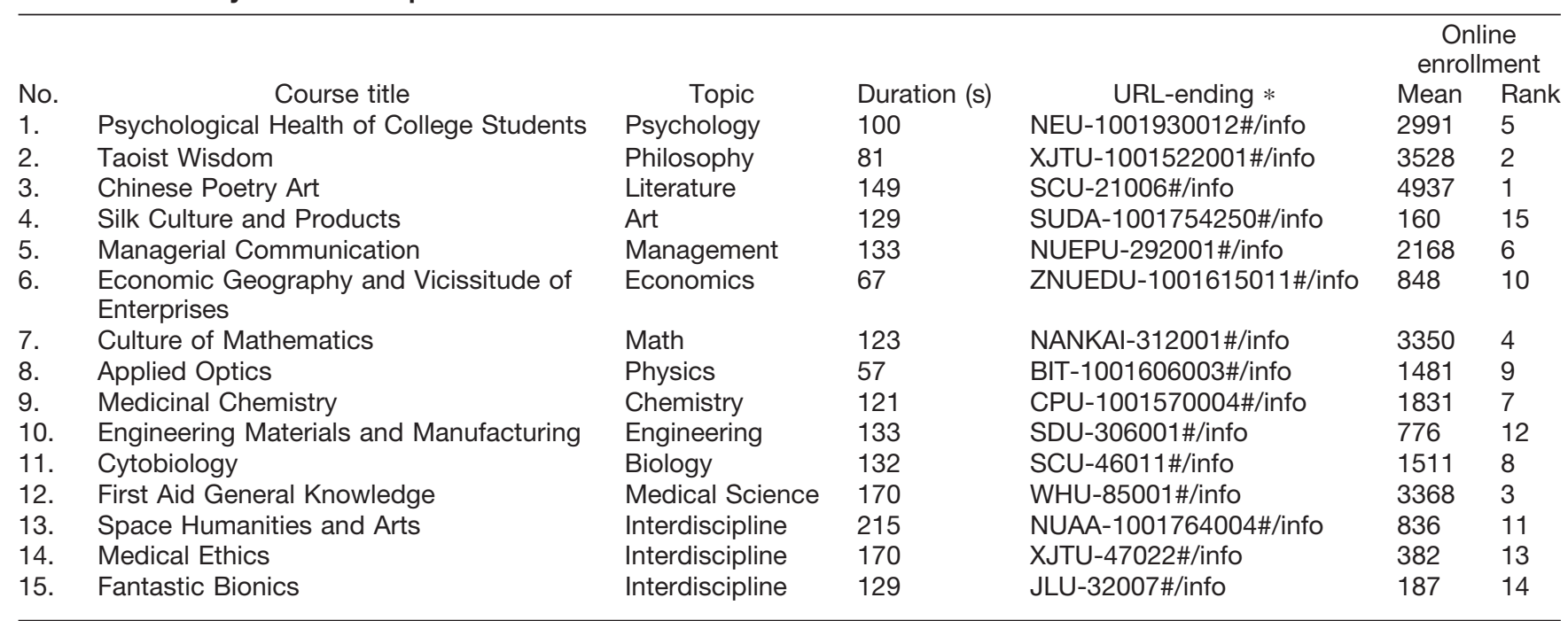

On-line enrollment (person-time/session) was recorded by the date of 2017/03/26.

*URL beginning with http://www.icourse163.org/course/.

to-normal vision and no history of neurological or psychiatric disorders. Monetary compensation was afforded for their participation. The study was approved by the Committee on Human Research Protection of East China Normal University (HR 064-2017). All subjects provided a written, signed informed consent form prior to the experiment.

\section{Materials}

Fifteen courses from Massive Open Online Courses (http://www.icourse163.org) were selected based on the following three criteria: (1) being designed by National Key Universities to ensure the production quality; (2) covering various topics in humanities, social sciences, and natural sciences; and (3) on-line enrollments of those courses were various (see details in Table 1). We focused and selected the introductory parts of those several-hour online video courses (https://www.icourse163.org/course/ WHU-85001), since the initial learning phase exerts an important effect on learning desire (Visser, 1998; Keller and Suzuki, 2004). The selected course clips were then edited (i.e., $1 \mathrm{~s}$ fade-out; resolution, $1280 \times 720$ ) using Movie Maker (Windows, Microsoft). The duration of each course clip lasted for $\sim 120 \mathrm{~s}$ (mean $\pm \mathrm{SD}, 127 \pm 41 \mathrm{~s}$; range, $57-215 \mathrm{~s}$ ).

\section{Procedures}

During the experiment, subjects were individually seated in front of a 19.5 inch monitor in an electromagnetic-soundshielded room, and wore earphones and an EEG recording cap (Fig. 1A).

There were 15 trials corresponding to 15 course clips. One trial entailed the following steps. First, a course title together with its preassigned course number (Table 1, see details) appeared for $3 \mathrm{~s}$, followed by a $1 \mathrm{~s}$ fixation. Next, subjects watched a course clip. After that, subjects provided answers to "Do you like the introduction?" and "Do you want to learn the course?" (1-100, from "not at all" to "very much," until response; Fig. 1B). Controlled by E-prime software (version 2.0; Psychology Software Tools), the presentation order of trials (course clips) was randomized across subjects.

Upon finishing 15 trials, 15 course titles with their course numbers were presented together on the screen. Subjects were then instructed to rank the 15 courses in order of their learning desire from 1 (most) to 15 (least). To do so, subjects wrote down corresponding course numbers beside a column of rankings (1-15) using a paper and pen (Fig. 1C). Upon finishing the course ranking, subjects were asked to rate the potential reasons to which they attributed their high learning desire on a 4-point scale from 1 (strongly disagree) to 4 (strongly agree). Two items testing the most concerned reasons, value and interest, were included: "learning the introduced course is useful for me" (Hulleman et al., 2010) and "I am interested in the introduced course" (Nuutila et al., 2018). As suggested by the precollected data from independent raters (see Statistical analyses), subjects reported reasons only for their top two courses (i.e., those were ranked with 1 and 2). To note, learning desire rankings of courses were later coded in reverse (i.e., 16 minus original rank), such that larger rankings indicated higher course-learning desire.

\section{EEG data acquisition and preprocessing}

Brain signals were recorded via a 64-channel EEG apparatus (Compumedics NeuroScan), in accordance with the international 10/10 system. The electrooculograms (EOGs) were recorded via four auxiliary electrodes. Two horizontal electrodes were placed lateralized to two eyes, while the other two vertical electrodes were placed over the upper and lower sides of the left eye. Data collected from the two horizontal electrodes and the two vertical electrodes were synthesized respectively and merged into one horizontal channel and one vertical channel. Impedances were kept to $<10 \mathrm{k} \Omega$. Signals were digitized at a sampling rate of $1000 \mathrm{~Hz}$. 
A

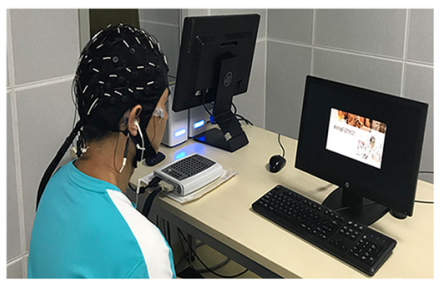

B

\section{C}

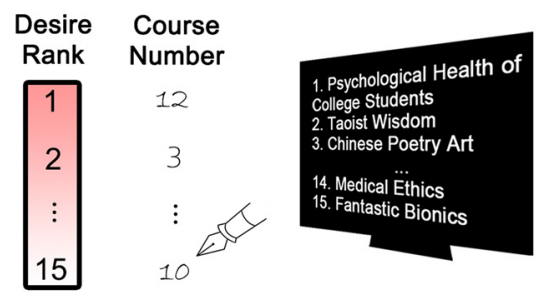

$\times 15$

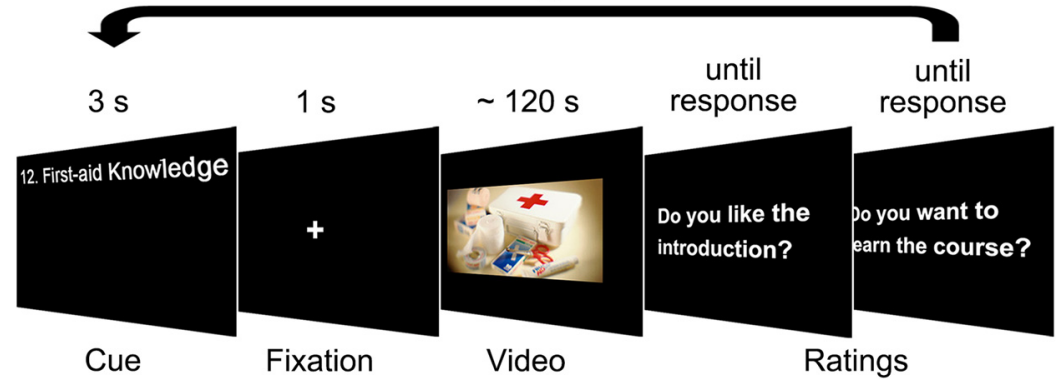

Figure 1. Schematic illustration of the experimental procedure. A, Experimental setup. $\boldsymbol{B}$, Events and time flows in a trial. $\boldsymbol{C}$, Subjects ranked courses based on their learning desire form 1 (highest) to 15 (lowest). Note that in the following analyses, rankings were reversely coded.

Following the study by Dmochowski et al. (2012), preprocessing of EEG data was performed using custom MATLAB (R2016b, MathWorks) scripts with EEGLAB toolbox (version 14.1.0; Delorme and Makeig, 2004). Data were filtered at a $1 \mathrm{~Hz}$ high-pass and a $50 \mathrm{~Hz}$ notch, and downsampled to $250 \mathrm{~Hz}$. As we focused on the EEG activity during the watching of course clips, data were segmented from the beginning to the end of each video. Eye-movement artifacts were corrected using a regression-based approach (Gratton et al., 1983; Elbert et al., 1985), as follows: (1) approximating the amplitude relation between EOG channels and each EEG channel and (2) then subtracting the estimated proportion of the EOG from the EEG. The regression-based correction was separately performed on the entire data block corresponding to each video. Two EOG channels and two mastoid channels were then omitted, leaving 60 channels in the following analyses. Bad channels were automatically rejected for exceeding mean channel power by 5 SDs. Outlier samples in each kept channel were rejected for their magnitudes exceeding the mean of that channel by more than 5 SDs. Data within -40 to $+40 \mathrm{~ms}(20$ sampling points) relative to each identified artifactual outlier were additionally rejected, and all were replaced by zeros. The preprocessed EEG data entered into subsequent analyses.

\section{Intersubject correlation}

The analysis of ISC (Dmochowski et al., 2012, 2014; see more details at www.parralab.org/isc/) was computed to quantify the neural similarity while subjects were watching the same naturalistic stimuli. It aims to find components (here, linear combinations of electrodes) capturing maximal correlation across all subjects. The concept of maximizing correlations resembled that in canonical correlation analysis (Hotelling, 1936), with a constraint being that the same projection vectors were used for all the datasets.

ISC analysis was performed individually for each course clip. It included three steps (Fig. 2). First, correlated components were captured across all subjects' neural datasets (subjects $\times$ electrodes $\times$ time-points) by solving an eigenvalue problem similar to the principle component analysis (Parra and Sajda, 2003). Second, the three strongest correlated components (i.e., C1, C2, and C3) were retained while other smaller ones were ignored (Dmochowski et al., 2012, 2014; Ki et al., 2016). Spatial distributions of the $\mathrm{C} 1, \mathrm{C} 2$, and $\mathrm{C} 3$, informing the approximate locations of the underlying neuronal sources, were visualized (Parra et al., 2005; Haufe et al., 2014). Finally, for each subject, component-wise (i.e., projected EEG data) correlations were computed between this subject and each of all remaining subjects, and then averaged. The ISC was then obtained as the sum of the correlation coefficients over $\mathrm{C} 1, \mathrm{C} 2$, and $\mathrm{C} 3$.

\section{Statistical analyses}

Following previous EEG-ISC studies (Dmochowski et al., 2012; Ki et al., 2016), we used a phase randomization technique to determine the chance-level ISC of each course clip (Theiler et al., 1992). To do so, fast Fourier transformation was first used to extract the original phases and amplitudes of preprocessed EEG data. Then, randomly generated phases were added to the original phases. With unchanged original amplitudes, inverse fast Fourier transformation was then used to reconstruct phase-randomized surrogate EEG data. By this step, the phase-randomized EEG surrogate data were not supposed to correlate across subjects. For each course clip, 


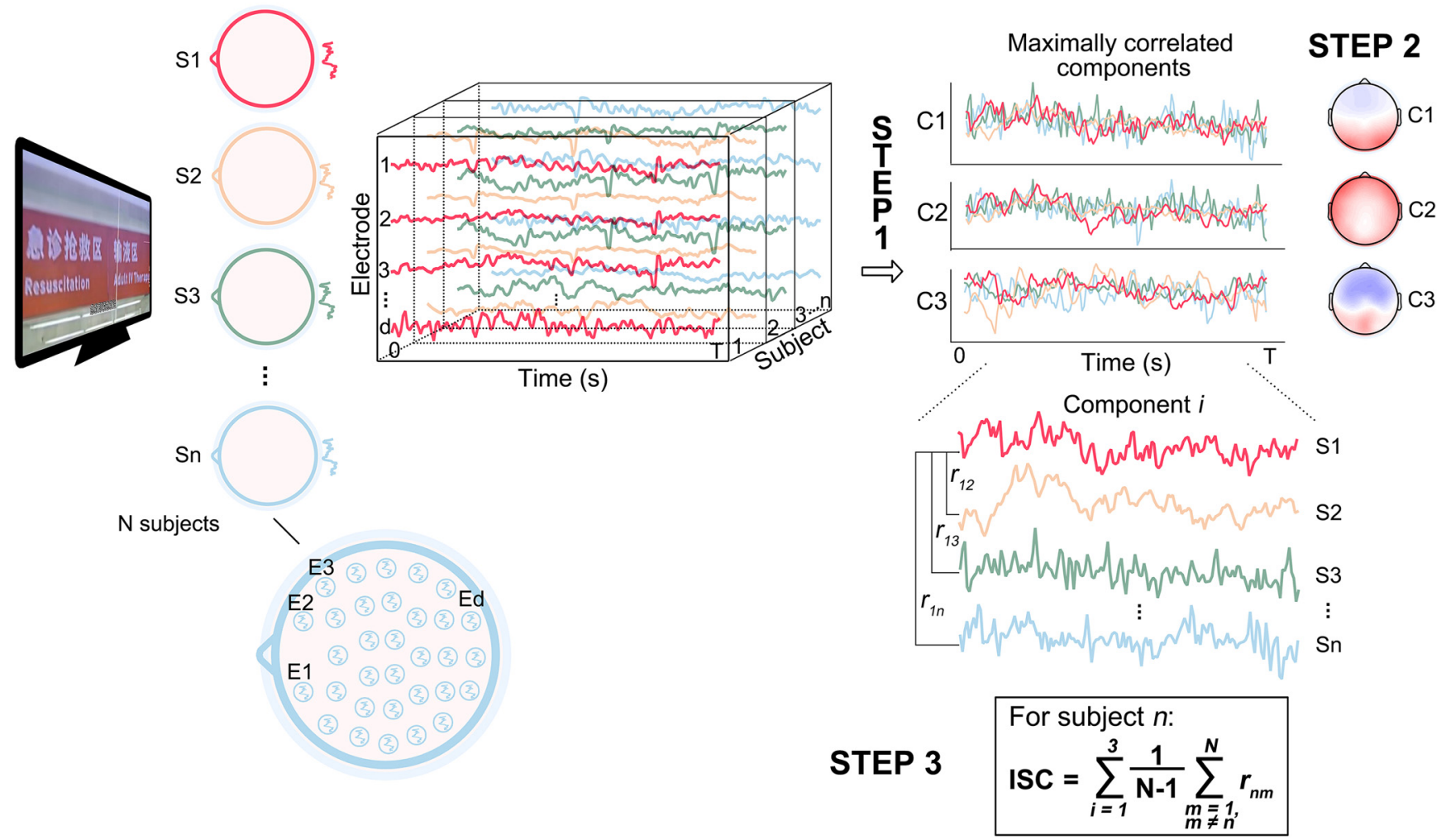

Figure 2. Overview of the three-step ISC analysis. Neural responses are recorded on $D$ electrodes from $N$ subjects during the time $(0-T \mathrm{~s})$ of stimuli presentation. First, a few (first three in this study) maximally correlated components are extracted. Second, the spatial distribution of each component is visualized. Third, for each subject, ISC is measured as the sum of the averaged correlation coefficients between that subject and remaining subjects over the first three components. Ed, Electrode $d$; Sn, subject $n$. Ci, component $i$.

the significance level was determined by comparing the ISC of the original data to the mean ISC of 1000 phaserandomized surrogate datasets. The resulting $p$ values for 15 course clips were then corrected using the false discovery rate (FDR) procedure (Benjamini and Yekutieli, 2001).

We calculated the motivational effectiveness of course clips by averaging the learning desire rankings across subjects. Fifteen course clips were then classified into three categories with different degrees of motivational effectiveness, high (average rankings from 11 to 15) versus medium (6-10) versus low (1-5); two clips (i.e., no. 3 and no. 12) were classified into the high effective category, 2 (i.e., no. 10 and no. 13) into the low effective category, and 11 (i.e., the remaining) into the medium effective category. Such a classification was validated by an additional group of independent raters. Prior to the EEG experiment, using the identical experimental procedures except for the EEG collection, behavioral data were precollected from an independent group of 25 subjects (six males; mean age, 21 years; age range, 19-25 years; 1 subject was left handed). The independent raters classified exactly the same 2 clips into the high effective category, exactly the same 2 into the low effective category, and exactly the same remaining 11 into the medium effective category as the EEG group did. To validate the use of group-averaged rankings for classification, we measured the variability of learning desire rankings for course clips across subjects (including EEG subjects and independent raters) using intraclass correlation (ICC). The ICC reached 0.93 , suggesting that the variability of the group-averaged rankings for course clips across subjects were fairly low (Koo and Li, 2016).

With the aforementioned classification of course clips, we then conducted one-way repeated-measures ANOVAs to relating motivational effectiveness (high vs medium vs low) with ISC values (i.e., ISC and subcomponents). Specifically, for each subject, ISC values of each effective category were first averaged across course clips in that category and then compared using repeated-measures ANOVAs, with motivational effectiveness (high vs medium vs low) as a within-subject variable. For post hoc pairwise comparisons, we used paired $t$ tests with FDR correction.

We further conducted a Pearson correlation analysis between ISC difference (high vs low) and learning desire difference (high vs low). Difference values (i.e., ISC difference and learning desire difference) were calculated by subtracting values of the low effective category (after averaging values across two involved course clips) from values of the high effective category (after averaging values across two involved course clips). To note, considering that ISC varies due to individual differences (Petroni et al., 2018), we chose to use the difference values rather than values of either low or high effective category. The ISC of the low effective category served as an active baseline and was subtracted to control for individual dif- 


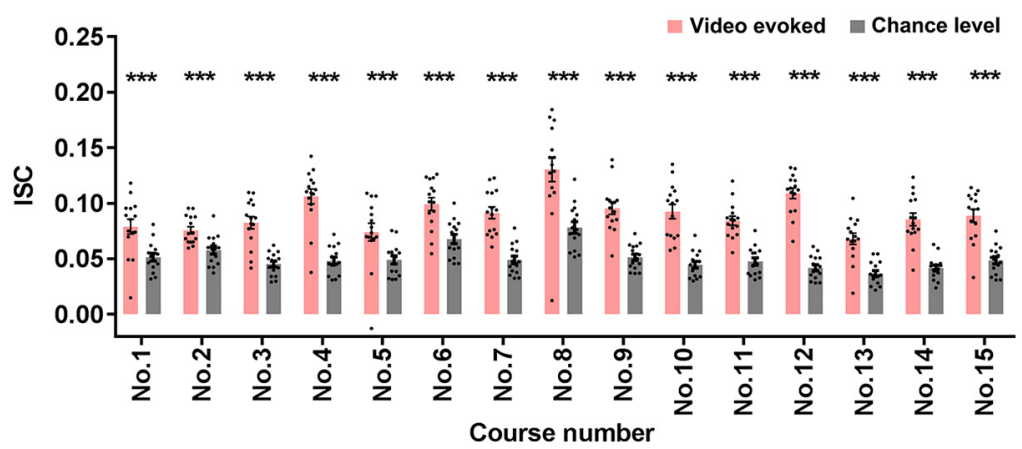

Figure 3. Video-evoked versus chance-level ISC of each course clip. ISC evoked by each course clip significantly exceeded its chance level. Each dot represents one subject. Error bars indicate SEs. $* * * p<0.001$, FDR corrected.

ferences. Here we focused on learning desire rankings decided after all the viewings of course clips rather than learning desire ratings of "do you want to learn the course?" collected after each course clip viewing since they were highly correlated with each other $\left(r_{(15)}=0.97, p\right.$ $<0.001)$ and the former were less biased to limited information. Given the evidence linking subcomponents of ISC (i.e., C1, C2, and C3) to separate cognitive functions (Dmochowski et al., 2014; Cohen and Parra, 2016), parallel correlation analyses were also separately performed between subcomponent ISCs difference (high vs low) and learning desire difference (high vs low).

Previous studies have found that human brain is optimized to make the fastest decision at a specified accuracy after successively integrating external perceptional inputs (Bogacz et al., 2006; Gold and Shadlen, 2007; de Gardelle and Summerfield, 2011; Tsetsos et al., 2012). How early brain responses predict subsequent behaviors has been computed by identifying the earliest time-point at which time-cumulative brain activity was significantly correlated with subsequent behaviors (Jiang et al., 2015; Zheng et al., 2018; Liu et al., 2019). Accordingly, we explored how early ISC predicted learning desire by identifying the earliest time-point, at which time the cumulative ISC difference (high vs low) was correlated with subsequent learning desire difference (high vs low). Specifically, time course correlation analyses between time-cumulative ISC difference and subsequent learning desire difference were repeatedly performed from 0.1 to $133 \mathrm{~s}$ (the shortest duration among the four course clips involved in low and high effective categories) with a time increment of $0.1 \mathrm{~s}$. The time-cumulative ISC at a certain cumulative time (ct) was computed by the time points from 1 to $c t \times$ 250 (sampling rate). The subsequent learning desire difference used in the time course correlation analyses was same as that used in the aforementioned full time correlation analysis. The resulting $p$ values, at the same size of repeated times for correlation analyses, were then corrected using FDR methods. Accordingly, if there existed a certain time point after which $p$ values of the correlations between the time-cumulative ISC difference and learning desire difference started and were maintained to survive the FDR correction, such a time-point would be labeled as the starting time point that ISC could successfully predict learning desire. Time increments of $0.5,1,2,5$, and $10 \mathrm{~s}$ also returned similar results. In addition, parallel time course correlation analyses were performed separately for subcomponent ISCs (C1, C2, and C3).

Moreover, we attempted to provide neurophysiological suggestions for why some naturalistic educational materials elicited high learning desire. To do so, for individual subjects, we focused on reason ratings (i.e., value and interest) and ISC of their own top two course clips with the highest rankings. Value and interest ratings were averaged across individual-level top two course clips and compared using a paired $t$ test. Next, we conducted Spearman correlation analyses between reason ratings (i.e., interest and value) and ISC, which was also averaged across individual-level top two course clips for each subject.

\section{Code accessibility}

The code described in the article is freely available on-line at https://github.com/YiZhuECNU/EEG-ISC.git. The code is available as Extended Data 1. It can be performed using MATLAB (version 2016b) in a Windows 10 system.

\section{Results}

\section{The significant ISCs for course clips}

As expected, each of 15 video-evoked ISCs (i.e., the averaged ISC across all the subjects) significantly exceeded its corresponding chance-level ISC determined by phase-randomized surrogated data ( $p$ values $<0.001$, FDR corrected; Fig. 3), indicating that course clips induced a significant learner-wise similar neural activities.

\section{ISC of high versus medium versus low effective course clips}

We next sought to identify whether ISC varied by motivational effectiveness. A one-way repeated-measures ANOVA comparing the ISC across motivational effectiveness (high vs medium vs low) factor on ISC was conducted. Results revealed a significant main effect $\left(F_{(2,28)}=\right.$ 8.36, $\left.p=0.001, \eta_{p}^{2}=0.37\right)$. Follow-up pairwise comparisons showed that the ISC was significantly larger for the medium effective category (mean $\pm \mathrm{SD}, 0.09 \pm 0.02$; $t_{(14)}$ $=3.18$; corrected $p<0.05$; Cohen's $d=0.68$ ) and for the high effective category $\left(0.10 \pm 0.02 ; t_{(14)}=3.25\right.$; corrected $p<0.05$; Cohen's $d=0.86$ ) than that for the low effective category $(0.08 \pm 0.02$; Fig. $4 A)$. 
A

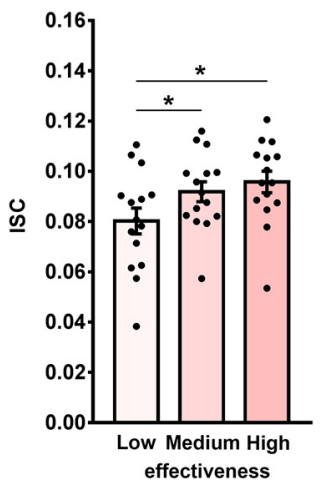

B

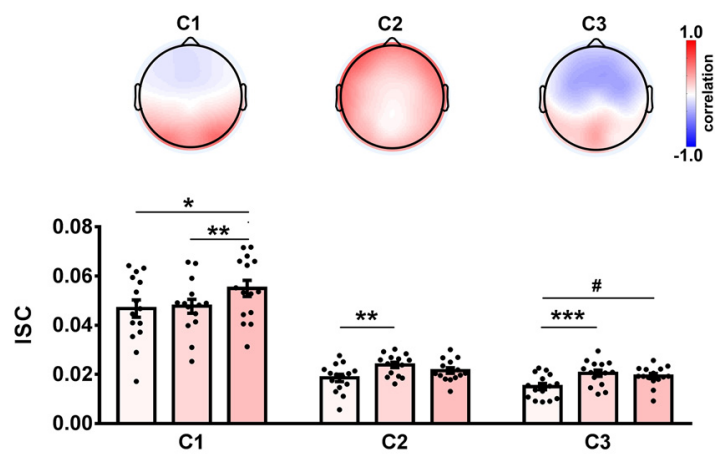

Figure 4. ISC of high versus medium versus low effective course clips. $\boldsymbol{A}$, ISCs of high and medium effective clips were respectively larger than that of low ones. $\boldsymbol{B}$, Top, One representative illustration (i.e., the highest effective course clip) of the scalp projections of the first three maximally correlated components (i.e., C1, C2, and C3). Color indicates how strongly the component activity correlated with the EEG signals recorded on different electrodes across the scalp. Bottom, Subcomponent ISCs were also enhanced when the motivational effectiveness of course clips increased. Each dot represents one subject. Error bars indicate SEs. \#p $<0.1, * p<0.05$, $* * p<0.01, * * * p<0.001$, FDR corrected.

Similar analyses on subcomponent ISCs (C1, C2, and C3) consistently showed the main effect of motivational effectiveness $\left(F\right.$ values $>6.78 ; p$ values $<0.004 ; \eta_{p}^{2}>$ 0.33). Follow-up pairwise comparisons showed the following results: for $\mathrm{C} 1$, the ISC of the high effective category was larger than that of the medium effective category $\left(t_{(14)}=3.98 ; p<0.01\right.$; Cohen's $\left.d=0.58\right)$ and that of the low effective category $\left(t_{(14)}=3.03 ; p<0.05\right.$; Cohen's $d=$ 0.59 ); for $\mathrm{C} 2$, the ISC of the medium effective category was significantly larger than that of the low effective category $\left(t_{(14)}=4.00 ; p<0.01\right.$; Cohen's $\left.d=0.98\right)$; for C3, the respective ISC of the medium and high effective categories was larger $\left(t_{(14)}=6.28 ; p<0.001\right.$; Cohen's $d=$ 1.01) and tended to be larger than that of the low effective category $\left(t_{(14)}=2.58 ; p<0.1\right.$; Cohen's $d=0.89$; Fig. $4 B$, bottom).

Representative spatial projections of three correlationmaximizing components on the scalp showed that the first component was symmetric and marked approximately in the frontal and occipital lobes, the second component was approximately in the bilateral frontotemporal lobes, and the third component was marked widely in the parietal cortex (Fig. 4B, top). Such scalp projections resulted from viewing course videos that were in accordance with those previously found for other audiovisual stimuli (Dmochowski et al., 2012, 2014).

\section{ISC as a predictor of course-learning desire}

We then tested whether the ISC predicted subjects' course-learning desire. ISC difference (high vs low) was significantly correlated with learning desire difference (high vs low: $r_{(15)}=0.74, p=0.002$; Fig. $5 A$ ). Parallel correlation analyses for subcomponent ISCs found that the difference in subcomponent ISCs (high vs low) was independently correlated with the learning desire difference (high vs low: $\mathrm{C} 1, r_{(15)}=0.66, p=0.007 ; \mathrm{C} 2, r_{(15)}=$ $0.58, p=0.02$; $C 3, r_{(15)}=0.47, p=0.08$; Fig. $5 C$ ).

To identify how an early ISC predicted learning desire, time course correlation analyses were repeatedly con- ducted with a $0.1 \mathrm{~s}$ time increment from 0.1 to $133 \mathrm{~s}$ between time-cumulative ISC difference (high vs low) and subsequent learning desire difference (high vs low). We found that at $100.6 \mathrm{~s}$ after the video onset, the timecumulative ISC difference (high vs low) started to become a significant predictor of subsequent learning desire difference (high vs low: $p<0.05$, FDR corrected; Fig. 5B). Later on, correlations remained constantly significant until the video ended. Parallel time course correlation analyses were conducted for each subcomponent ISC. Results revealed a key role of $\mathrm{C} 1$ (but not of $\mathrm{C} 2$ and $\mathrm{C} 3$ ) in the prediction (starting at $85.5 \mathrm{~s}$; Fig. $5 D$ ).

\section{The association between ISC and interest/value}

In an attempt to provide neurophysiological suggestions for why some course clips are effective to evoke learning desire, we tested whether ISC was associated with value and/or interest. Behaviorally, ratings of interest (mean $\pm \mathrm{SD}, 3.67 \pm 0.36$ ) were highest among all the reasons and significantly exceeded ratings of value (3.27 $\pm 0.62 ; t_{(14)}=2.45, p=0.03$, Cohen's $d=0.79$; Fig. $6 A$ ). Moreover, the ISC for individual-level top two course clips was significantly correlated with ratings of interest $\left(r_{(15)}=\right.$ $0.77, p=0.0008$; Fig. $6 B)$, but not of value $\left(r_{(15)}=0.32, p\right.$ $=0.25$; Fig. 6 C).

\section{Discussion}

Here we used an EEG-derived ISC approach to capture the degree of shared brain responses to naturalistic educational materials. Results revealed that (1) on-line course videos prompted similar neural activity across learners; (2) the neural similarity was enhanced by the motivational effectiveness of course clips for evoking learning desire; (3) the neural similarity was predictive of course-learning desire (even before finishing the viewing of the entire video); and (4) the neural similarity was associated with interest-based (rather than value-based) learning desire. These results are discussed successively as follows. 
A

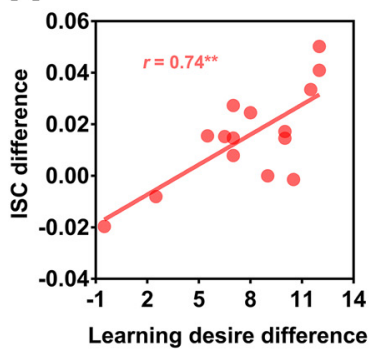

C

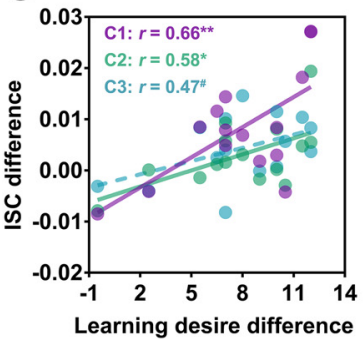

B

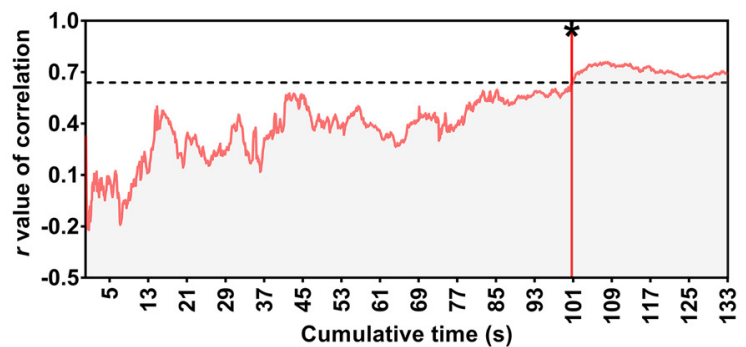

D

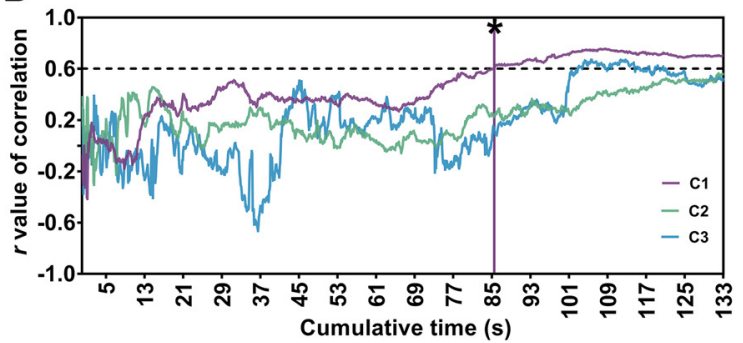

Figure 5. ISC predicted course-learning desire. A, Pearson correlation indicated ISC difference (high vs low) positively associated with the subject's learning desire difference (high vs low; $r_{(15)}=0.74, p=0.002$ ). $\boldsymbol{B}$, ISC difference became a significant correlate of learning desire difference after $\sim 100 \mathrm{~s}$ of watching. The vertical red line with an asterisk indicates the earliest time (100.6 s) at which such a correlation reached the significance. The horizontal dashed line indicates the correlation coefficient $\left(r_{(15)}=0.64, p<0.05\right.$, FDR corrected). C, Pearson correlations indicated differences in subcomponent ISCs independently associated with the subject's learning desire difference. $\boldsymbol{D}$, For $\mathrm{C} 1$, the vertical purple line with an asterisk indicates the earliest time (85.5 s) at which correlation reached the significance. The horizontal dashed line indicates the correlation coefficient $\left(r_{(15)}=0.60, p<0.05\right.$, FDR corrected). C2 or C3 showed no such early prediction effect. Each dot represents one subject. $\# p<0.1, * p<0.05, * * p<0.01$.

First, using EEG, we recorded learners' general patterns of neuronal activity at the timescale during the watching of on-line course videos. We found that all 15 course clips, regardless of their motivational effectiveness for evoking learning desire, elicited significant neural similarity across learners. This result aligns well with pervious findings that brains of different individuals tend to act in unison during the natural watching (Hasson et al., 2004; Dmochowski et al., 2012, 2014; Ki et al., 2016). Thus, an ISC across multiple brains tends to provide a sensitive and quantifiable measure of the continuous neural responses to naturalistic stimuli. Critically, this measure makes it feasible for conventional laboratory paradigms to move beyond the rigid trial-based structure where discrete stimuli are repetitively presented.
Second, although all course clips prompted similar neural processing across learners, we found significantly larger ISC for high (vs medium, vs low) effective course clips. It seems that course clips, which engage learners' brains more collectively, are more effective to evoke course-learning desire. This finding is consistent with prior studies demonstrating larger ISC during strong (vs weak) powerful political speeches (Schmälzle et al., 2015), and more (vs less) effective antialcohol public service announcements (Imhof et al., 2017). An ISC has also been found to predict the preferential effectiveness of advertisements in an EEG study (Dmochowski et al., 2014). However, here we failed to demonstrate that ISC scaled monotonically with the motivational effectiveness of course clips. To note, the duration of advertisements used
A

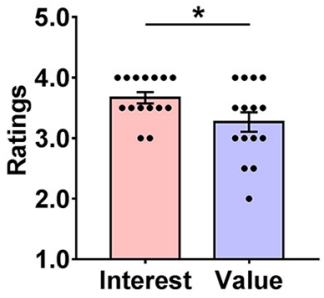

B

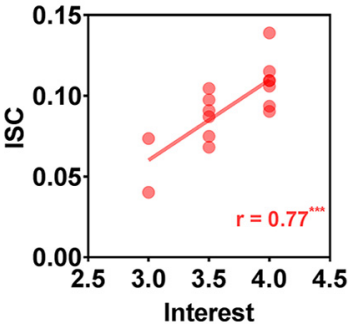

C

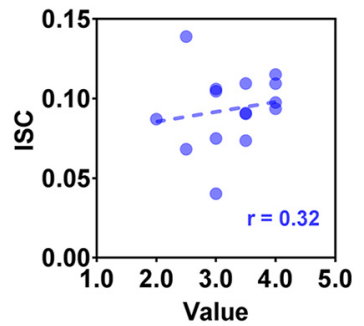

Figure 6. ISC was associated with interest rather than value. $\boldsymbol{A}$, Ratings of interest significantly exceeded those of value. $\boldsymbol{B}, \boldsymbol{C}$, For individual-level high effective course clips, the Spearman correlation indicated that ISC positively associated with the ratings of value $\left(r_{(15)}=0.77, p=0.0008 ; \boldsymbol{B}\right)$, but not with ratings of interest $\left(r_{(15)}=0.32, p=0.25 ; \boldsymbol{C}\right)$. Each dot represents one subject. Error bars indicate SEs. $* p<0.05, * * * p<0.001$. 
in the study by Dmochowski et al. (2014) is identical (i.e., $30 \mathrm{~s}$ ), while the duration of course clips used in our study is not (i.e., 57-215 s). We suspected that the longer watching of materials might damage the sustained attention or vigilance (Nuechterlein et al., 1983; Sarter et al., 2001), thence modulating the ISC (Ki et al., 2016; lotzov et al., 2017; Cohen et al., 2018).

Third, course-learning desire could be predicted by the neural similarity. Moreover, time course analyses showed that ISC was predictive of subsequent course-learning desire after $\sim 80 \mathrm{~s}$ of watching of videos. The first maximally correlated component (C1) played a key role in such a prediction. Representative scalp projection of the $\mathrm{C} 1$ exhibited a symmetric pattern marked in the frontal and occipital electrodes. Such a component captures the neural activity possibly reflecting the visual processing (Dmochowski et al., 2012, 2014), suggesting that the visual property of educational materials is crucial for promoting learning desire. This finding is in accordance with those of previous studies showing that visual properties, such as saliency, influence the final decision (Milosavljevic et al., 2012; Towal et al., 2013). The second component (C2) in the bilateral frontotemporal lobes was possibly recruited in the auditory processing (Hickok and Poeppel, 2007). Besides, C1 and C2 might also capture supramodal responses (Cohen and Parra, 2016). The third component (C3) was marked widely in the parietal cortex, which might be associated with attentional modulation to audiovisual stimuli (Shomstein and Yantis, 2006; Nardo et al., 2011). The global scalp patterns observed in the current study aligned with those found in a previous fMRI study, where spatial dimension of the observed EEG-derived neural similarity was probed by regressing BOLD activation time series onto the neural similarity scores (Dmochowski et al., 2014). In a final detail, $\sim 80 \mathrm{~s}$ of video watching was sufficient to predict the course-learning desire. This finding bolsters the optimization of brain to make the fastest decision at a specified accuracy after successively integrating external perceptional inputs (Bogacz et al., 2006; Gold and Shadlen, 2007; de Gardelle and Summerfield, 2011; Tsetsos et al., 2012).

Fourth, we provided neurophysiological suggestions for why some course clips were effective to evoke learning desire by testing the association between ISC and value/ interest. For course clips ranked with higher learning desire by individuals, (1) interest was reported to be a more important reason for further course study, and (2) neural similarity during the processing of those videos was associated with self-reported interest rather than value. These findings support interest-based learning desire hypothesis-learners' interests effectively promote learning desire (Ainley et al., 2002; Harackiewicz et al., 2002; Krapp, 2002; Hidi and Renninger, 2006). Given the evidence that ISC is strongly modulated by attention ( $\mathrm{Ki}$ et al., 2016; lotzov et al., 2017; Cohen et al., 2018) and the potential association between attention and interest (Shirey, 1992), we suspected that attention might play a role in the relationship between ISC and interest-based learning desire. As an important note, although valuebased learning desire hypothesis did not gain the sup- porting results in the current study, we could not assuredly draw a conclusion that value played no role in promoting learning desire. It might be the case that our ISC measure was not so sensitive to the value-based on-line learning. Future independent replications are needed to provide more evidence.

Several limitations of this work, along with future directions, deserve to be noted. First, scalp projections of correlated components are not valid to exactly locate brain sources due to the inherently limited spatial resolution of EEG. Therefore, future studies should consider adopting source analyses (e.g., standardized lowresolution brain electromagnetic tomography; PascualMarqui, 2002) with high-resolution EEG, as well as fMRI/ MEG with satisfactory spatial resolution (Dmochowski et al., 2014). Second, post hoc power analyses with G*Power (Erdfelder et al., 1996) indicated that a sample size of $\sim 13$ would be sufficient to obtain statistical power at the recommended 0.8 level (Cohen, 1988) with Cohen's $d=0.86$ reported in the result that ISC was larger for high (vs low) effective course clips. However, our sample size $(N=15)$ was far from adequate to examine how individuals' factors (e.g., goal orientation; Elliot and Mcgregor, 2001) influenced the neural responses to educational messages in a top-down manner, calling for future studies. Finally, value and interest were viewed independently in the present study, since the correlation conducted on individual subjects between value and interest ratings for their own high effective course clips was not significant $\left(r_{(15)}=0.26, p=0.34\right)$. However, value and interest have been found to interact with each other and have interplay effects on competence belief, success expectancy, and learning performance (Hidi and Renninger, 2006; Fryer and Ainley, 2018; Nuutila et al., 2018). Future studies might test the interplay of value and interest in other contexts of on-line learning [e.g., courses with (1) high value + high interest, (2) high value + low interest, (3) low value + high interest, and (4) low value + low interest, and the power of ISC measure to differentiate between them].

To sum up, the current results indicate that naturalistic educational materials with greater motivational effectiveness enhanced neural similarity across learners. Such enhanced neural similarity is predictive of learning desire, which is based on interest. From a collective-brain perspective, the use of EEG-derived ISC approach holds the potential to evaluate the motivational effectiveness of naturalistic educational materials. Our study paves the way to investigate learners' motivation at a neurophysiological level in the context of on-line learning. It also holds relevance for instructional designs to aid learning interest deficit.

\section{References}

Ainley M, Hillman K, Hidi S (2002) Gender and interest processes in response to literary texts: situational and individual interest. Learn Instr 12:411-428.

Atkinson JW (1957) Motivational determinants of risk-taking behavior. Psychol Rev 64:359-372.

Benjamini Y, Yekutieli D (2001) The control of the false discovery rate in multiple testing under dependency. Ann Stat 1165-1188. 
Bogacz R, Brown E, Moehlis J, Holmes P, Cohen JD (2006) The physics of optimal decision making: a formal analysis of models of performance in two-alternative forced-choice tasks. Psychol Rev 113:700-765.

Canning EA, Harackiewicz JM, Priniski SJ, Hecht CA, Tibbetts Y, Hyde JS (2018) Improving performance and retention in introductory biology with a utility-value intervention. J Educ Psychol 110: 834-849.

Cohen J (1988) Statistical power analysis for the behavioral sciences. San Diego, CA: Academic.

Cohen SS, Parra LC (2016) Memorable audiovisual narratives synchronize sensory and supramodal neural responses. eNeuro 10: ENEURO.0203-16.2016.

Cohen SS, Henin S, Parra LC (2017) Engaging narratives evoke similar neural activity and lead to similar time perception. Sci Rep 7:4578.

Cohen SS, Madsen J, Touchan G, Robles D, Lima SF, Henin S, Parra LC (2018) Neural engagement with online educational videos predicts learning performance for individual students. Neurobiol Learn Mem 155:60-64.

Copley J (2007) Audio and video podcasts of lectures for campusbased students: production and evaluation of student use. Innov Educ Teach Int 44:387-399.

De Gardelle V, Summerfield C (2011) Robust averaging during perceptual judgment. Proc Natl Acad Sci U S A 108:13341-13346.

Delorme A, Makeig S (2004) EEGLAB: an open source toolbox for analysis of single-trial EEG dynamics including independent component analysis. J Neurosci Methods 134:9-21.

Dmochowski JP, Sajda P, Dias J, Parra LC (2012) Correlated components of ongoing EEG point to emotionally laden attention-a possible marker of engagement? Front Hum Neurosci 6:112.

Dmochowski JP, Bezdek MA, Abelson BP, Johnson JS, Schumacher EH, Parra LC (2014) Audience preferences are predicted by temporal reliability of neural processing. Nat Commun 5:4567.

Eccles J, Adler TF, Futterman R, Goff SB, Kaczala CM, Meece JL, Midgley C (1983) Expectancies, values, and academic behaviors. In: Achievement and achievement motives: psychological and sociological approaches (Spence JT, ed), pp 75-146. San Francisco, CA: Freeman.

Elbert T, Lutzenberger W, Rockstroh B, Birbaumer N (1985) Removal of ocular artifacts from the EEG-a biophysical approach to the EOG. Electroencephalogr Clin Neurophysiol 60:455-463.

Elliot AJ, Mcgregor HA (2001) A 2 X 2 achievement goal framework. J Pers Soc Psychol 80:501-519.

Erdfelder E, Faul F, Buchner A (1996) GPOWER: a general power analysis program. Behav Res Methods Instrum Comput 28:1-11.

Fryer LK, Ainley M (2018) Supporting interest in a study domain: a longitudinal test of the interplay between interest, utility-value, and competence beliefs. Learning and Instruction, in press.

Gold JI, Shadlen MN (2007) The neural basis of decision making. Annu Rev Neurosci 30:535-574.

Grant MR, Thornton HR (2007) Best practices in undergraduate adult-centered online learning: mechanisms for course design and delivery. J Online Learn Teach 3:346-356.

Gratton G, Coles MG, Donchin E (1983) A new method for off-line removal of ocular artifact. Electroencephalogr Clin Neurophysiol 55:468-484.

Harackiewicz JM, Barron KE, Pintrich PR, Elliot AJ, Thrash TM (2002) Revision of achievement goal theory: necessary and illuminating. J Educ Psychol 94:638-645.

Hasson U, Nir Y, Levy I, Fuhrmann G, Malach R (2004) Intersubject synchronization of cortical activity during natural vision. Science 303:1634-1640.

Haufe S, Meinecke F, Görgen K, Dähne S, Haynes JD, Blankertz B, Bießmann $F$ (2014) On the interpretation of weight vectors of linear models in multivariate neuroimaging. Neuroimage 87:96-110.

Hidi S, Renninger KA (2006) The four-phase model of interest development. Educ Psychol 41:111-127.

Hickok G, Poeppel D (2007) The cortical organization of speech processing. Nat Rev Neurosci 8:393-402.
Hotelling $\mathrm{H}$ (1936) Relations between two sets of variates. Biometrika 28:321-377.

Hulleman CS, Durik AM, Schweigert SA, Harackiewicz JM (2008) Task values, achievement goals, and interest: an integrative analysis. J Educ Psychol 100:398-416.

Hulleman CS, Godes O, Hendricks BL, Harackiewicz JM (2010) Enhancing interest and performance with a utility value intervention. J Educ Psychol 102:880-895.

Imhof MA, Schmälzle R, Renner B, Schupp HT (2017) How real-life health messages engage our brains: shared processing of effective anti-alcohol videos. Soc Cogn Affect Neurosci 12:1188-1196.

Iotzov I, Fidali BC, Petroni A, Conte MM, Schiff ND, Parra LC (2017) Divergent neural responses to narrative speech in disorders of consciousness. Ann Clin Transl Neurol 4:784-792.

Jiang J, Chen C, Dai B, Shi G, Ding G, Liu L, Lu C (2015) Leader emergence through interpersonal neural synchronization. Proc Natl Acad Sci 112:4274-4279.

Kay RH (2012) Exploring the use of video podcasts in education: a comprehensive review of the literature. Comput Human Behav 28:820-831.

Keller J, Suzuki K (2004) Learner motivation and e-learning design: a multinationally validated process. J Educ Media 29:229-239.

Ki JJ, Kelly SP, Parra LC (2016) Attention strongly modulates reliability of neural responses to naturalistic narrative stimuli. J Neurosci 36:3092-3101.

Kizilcec RF, Bailenson JN, Gomez CJ (2015) The instructor's face in video instruction: evidence from two large-scale field studies. J Educ Psychol 107:724-739.

Koo TK, Li MY (2016) A guideline of selecting and reporting intraclass correlation coefficients for reliability research. J Chiropr Med 15: 155-163.

Krapp A (2002) Structural and dynamic aspects of interest development: theoretical considerations from an ontogenetic perspective. Learn Instr 12:383-409.

Liu J, Zhang R, Geng B, Zhang T, Yuan D, Otani S, Li X (2019) Interplay between prior knowledge and communication mode on teaching effectiveness: interpersonal neural synchronization as a neural marker. Neuroimage 193:93-102.

Milosavljevic M, Navalpakkam V, Koch C, Rangel A (2012) Relative visual saliency differences induce sizable bias in consumer choice. J Consum Psychol 22:67-74.

Mitchell M (1993) Situational interest: its multifaceted structure in the secondary school mathematics classroom. J Educ Psychol 85: 424-436.

Nardo D, Santangelo V, Macaluso E (2011) Stimulus-driven orienting of visuo-spatial attention in complex dynamic environments. Neuron 69:1015-1028.

Nuechterlein KH, Parasuraman R, Jiang Q (1983) Visual sustained attention: image degradation produces rapid sensitivity decrement over time. Science 220:327-329.

Nummenmaa L, Glerean E, Viinikainen M, Jaaskelainen IP, Hari R, Sams M (2012) Emotions promote social interaction by synchronizing brain activity across individuals. Proc Natl Acad Sci U S A 109:9599-9604.

Nuutila K, Tuominen H, Tapola A, Vainikainen MP, Niemivirta M (2018) Consistency, longitudinal stability, and predictions of elementary school students' task interest, success expectancy, and performance in mathematics. Learn Instr 56:73-83.

Parra LC, Sajda P (2003) Blind source separation via generalized eigenvalue decomposition. J Mach Learn Res 4:1261-1269.

Parra LC, Spence CD, Gerson AD, Sajda P (2005) Recipes for the linear analysis of EEG. Neuroimage 28:326-341.

Pascual-Marqui RD (2002) Standardized low-resolution brain electromagnetic tomography (sLORETA): technical details. Methods Find Exp Clin Pharmacol 24(Suppl D):5-12.

Petroni A, Cohen SS, Ai L, Langer N, Henin S, Vanderwal T, Milham MP, Parra LC (2018) The variability of neural responses to naturalistic videos change with age and sex. eNeuro 5:e0244-17.20. 
Poulsen AT, Kamronn S, Dmochowski J, Parra LC, Hansen LK (2017) EEG in the classroom: synchronised neural recordings during video presentation. Sci Rep 7:43916.

Renninger KA, Hidi S (2002) Student interest and achievement: developmental issues raised by a case study. In: Development of achievement motivation (Eccles AWJS, ed), pp 173-195. New York, NY: Academic.

Rotgans JI, Schmidt HG (2014) Situational interest and learning: thirst for knowledge. Learn Instr 32:37-50.

Sarter M, Givens B, Bruno JP (2001) The cognitive neuroscience of sustained attention: where top-down meets bottom-up. Brain Res Rev 35:146-160.

Schiefele U (2009) Situational and individual interest. In: Handbook of motivation at school (Wentzel KR, Wigfield A, eds), pp 197-222. New York: Routledge.

Schmälzle R, Häcker FE, Honey CJ, Hasson U (2015) Engaged listeners: shared neural processing of powerful political speeches. Soc Cogn Affect Neurosci 10:1137-1143.

Shirey LL (1992) Importance, interest, and selective attention. In: The role of interest in learning and development (Renninger KA, Hidi S, Krapp A, eds), pp 281-296. Hillsdale, NJ: Earlbaum.

Shomstein S, Yantis S (2006) Parietal cortex mediates voluntary control of spatial and nonspatial auditory attention. J Neurosci 26:435-439.

Spiers HJ, Maguire EA (2007) Decoding human brain activity during real-world experiences. Trends Cogn Sci 11:356-365.
Szpunar KK, Khan NY, Schacter DL (2013) Interpolated memory tests reduce mind wandering and improve learning of online lectures. Proc Natl Acad Sci U S A 110:6313-6317.

Theiler J, Eubank S, Longtin A, Galdrikian B, Farmer JD (1992) Testing for nonlinearity in time series: the method of surrogate data. Physica D 58:77-94.

Todd S (2013) Learning desire: perspectives on pedagogy, culture, and the unsaid. Hoboken, NJ: Taylor and Francis.

Towal RB, Mormann M, Koch C (2013) Simultaneous modeling of visual saliency and value computation improves predictions of economic choice. Proc Natl Acad Sci U S A 110:E3858-E3867.

Tsetsos K, Chater N, Usher M (2012) Salience driven value integration explains decision biases and preference reversal. Proc Natl Acad Sci U S A 109:9659-9664.

Updegraff KA, Eccles JS, Barber BL, O'brien KM (1996) Course enrollment as self-regulatory behavior: who takes optional high school math courses? Learn Individ Differ 8:239-259.

Visser $L$ (1998) The development of motivational communication in distance education support. Enschede, The Netherlands: Universiteit Twente.

Waldrop MM (2013) Campus 2.0. Nature 495:160-163.

Zheng L, Chen C, Liu W, Long Y, Zhao H, Bai X, Zhang Z, Han Z, Liu L, Guo T, Chen B, Ding G, Lu C (2018) Enhancement of teaching outcome through neural prediction of the students' knowledge state. Hum Brain Mapp 39:3046-3057. 